\title{
Erectile function after repeat saturation prostate biopsy: Our experience in 100 patients
}

\author{
Pietro Pepe, Francesco Pietropaolo, Giuseppe Dibenedetto, Francesco Aragona \\ Urology Unit, Cannizzaro Hospital, Catania, Italy.
}

\begin{abstract}
Г Summary
Introduction: Erectile dysfunction (ED) incidence following repeat saturation prostate biopsy (SPBX) was evaluated.

Materials and methods: From January 2011 to June 2012295 patients underwent repeat transperineal SPBX (median 28 cores) under sedation. The indications for biopsy were: abnormal DRE, PSA $>10 \mathrm{ng} / \mathrm{mL}$ or included between 4.1-10 with free/total PSA $<25 \%$. All patients were prospectively evaluated with the 5-item version of the International Index of Erectile Function (IIEF-5) at baseline and 1, 3 and 6 months from SPBX.

Results: 100/200 men with benign histology and normal sexual activity completed the study; median IIEF-5 score before and after SPBx was equal to 18.3 (baseline) vs 17.8 (1 month later) vs 18 (3 months later) vs 18.1 (6 months later) ( $p>0.05$ ); in detail, 1 month from biopsy 5 (5\%) men referred a mild ED that disappeared at 3 and 6 months evaluation.

Conclusions: Repeat transperineal SPBX under sedation did not significantly worsened erectile function; the minimal risk of temporary post-biopsy ED could be previously discussed (not emphasised) with potent patients.
\end{abstract}

KEY WORDS: Erectile dysfunction; Saturation prostate biopsy; Sexuality; Prostate biopsy.

Submitted 27 February 2013; Accepted 30 April 2013

No conflict of interest declared

\section{INTRODUCTION}

Prostate cancer ( $\mathrm{PCa}$ ) is the most frequent tumor diagnosed in elder men with about 1 million biopsies for year performed in the United States (1). In the last decade a greater rate of prostate biopsy side effects has been reported and serious complications incidence requiring hospital admission ranges from $1.2 \%$ (2) to $2.5 \%$ (1) secondary, in the most of the cases, to urinary tract infection (UTI), fever or sepsis (in case of transrectal biopsy). Among minor complications erectile dysfunction (ED) has been reported in a little percentage of patients and it has been ascribed to anxiety (3), local anesthesia and/or number of needle cores $(4,5)$ inducing a clinical impact on patient well-being and quality of life (6). In our study ED incidence following repeat transperineal saturation prostate biopsy (SPBx) was prospectively evaluated.

\section{MATERIALS AND MEthodS}

From January 2011 to June 2012295 patients of ages between 56 and 71 years (median 63 years) underwent repeat SPBx (median 28 cores; range: 26-31) for persistent suspicious of PCa. The indications for biopsy were (7): abnormal digital rectal examination (DRE), persistent elevated or increasing PSA values, PSA $>10 \mathrm{ng} / \mathrm{mL}$, PSA values between 4.1-10 with free/total PSA $<25 \%$. Prostate biopsy was performed transperineally (8) using a tru-cut 18 gauge needle (Bard; Covington, GA), a GE Logiq 500 PRO ecograph (General Electric; Milwaukee, WI) supplied with a biplanar transrectal probe (5-6.5 $\mathrm{MHz}$ ). The SPBx included at least 12 cores in the posterior zone of each lobe (apex, middle zone and base of the gland) beginning parasagittally to reach the outer edges of the gland (lateral margins) plus 2-3 cores in the transition and anterior zone. The procedure was performed under sedation and antibiotic prophylaxis, respectively. Among clinical complications incidence of ED was evaluated only in men with benign histology (normal parenchy$\mathrm{ma}$ ), on the contrary patients with cancer, ASAP or HGPIN were not included to eliminate anxiety from the hypothetical cause of ED. All patients were prospectively evaluated 
with the 5-item version of the International Index of Erectile Function (IIEF-5) (9) before and 1, 3 and 6 months from SPBx. None of the patients used 5-phosphodiesterase inhibitors or prostaglandins to improve sexual activity. For statistical analysis the t Student' - test was used; a p value $<0.05$ was considered statistically significant.

\section{RESULTS}

Histological specimen showed a PCa in 90/295 (30.5\%) cases, an HGPIN in 4 (1.3\%), an ASAP $1(0.4 \%)$ and a

Table 1.

Clinical findings in 100 patients who underwent repeat saturation prostate biopsy.

\begin{tabular}{|lc|}
\hline $\begin{array}{l}\text { Clinical findings } \\
\text { Median age (years) }\end{array}$ & $\begin{array}{c}\text { No (\%) of patients } \\
\text { 61 (range: 56-71) }\end{array}$ \\
\hline PSA 4.1-10 $\mathbf{~ g / m L ~}$ & 68 \\
PSA > 10 ng/mL & 32 \\
\hline Abnormal DRE & - \\
\hline LUTS & 67 \\
$\mathbf{Q}_{\text {max }}$ & $11(8-19)$ \\
IPSS (median) & $11(4-29)$ \\
\hline Comorbidities: & 18 \\
Diabetes mellitus & 3 \\
Hypertension & 10 \\
Gastritis & 18 \\
Other & 3 \\
\hline Drug therapy (overall): & 86 \\
Oral hypoglycemic & 2 \\
Antihypertensive & 10 \\
Antiplatelet agents & 10 \\
Diuretic & 3 \\
Proton pomp inhibitor & 19 \\
Alfa-blockers & 62 \\
Other & 6 \\
\hline
\end{tabular}

DRE: digital rectal examination; LUTS: lower urinary tract symptoms; IPSS: international prostate symptoms score. normal parenchyma in 200 (67.8\%), respectively; $100 / 200$ (50\%) men with a referred normal sexual activity characterized by a median baseline IIEF- 5 score equal to 18.3 (range: 16-25) completed the study. Clinical (comorbidities, drug therapy) and laboratory data collected before SPBx are reported in Table 1.

Among clinical complications none of the patients needed hospital admission and 10 (10\%) underwent emergency clinic visit within 2 day (median; range: 1-3 days) from SPBx in 7 cases (7\%) for acute urinary retention, in $2(2 \%)$ for gross hematuria and in 1 (1\%) for urinary tract infection; moreover, hemospermia was the most frequent side effect recorded in 36 (36\%) and 9 (9\%) patients 3 and 6 months from the procedure, respectively. A significantly difference between IEEF-5 score at baseline (18.3) and 1 (17.8), 3 (18) and 6 (18.1) months from SPBx was not found ( $>$ > 0.05) (Table 2); in detail, after 1 month 5 (5\%) patients with a pre-biopsy normal sexual activity (IIEF $>22$ ) referred a mild DE that disappeared at 3 and 6 months evaluation (Table 2).

\section{Discussion}

A minimal risk of temporary ED following prostate biopsy has been correlated with the increasing number of needle cores, use of periprostatic nerve block, disease involving neurovascular bundles, anxiety and diagnosis of PCa. Although Tuncel et al. (10) reported a male sexual dysfunction combined with negative effect on female sexual function exceeding six months from biopsy, most of the papers $(5,10)$ agree that prostate biopsies could have a significantly impact on short-term (30 days) erectile function that disappears at medium-term (3-6 months).

Glaser et al (3) in a systematic review reported that prostate biopsy was associated with short-term exacerbation of urinary symptoms score, anxiety and ED without a distinct relationship to the periprostatic nerve block or the number of cores biopsied. Zisman et al. (4) attributed to anxiety a ED rate in anticipation of biopsy in $7 \%$ of the cases evaluated; Akbal et al. (5) in 88 patients cancer-free after SPBx showed a risk of ED after 1 and 6 months

Table 2.

IIEF-5 (International Index Erectile Function) in 100 patient before (baseline) and after (1, 3 and 6 months) repeat transperineal saturation prostate biopsy.

\begin{tabular}{|c|c|c|c|c|c|}
\hline $\begin{array}{l}\text { IEFF-5 } \\
\text { (score: 5-25) }\end{array}$ & $\begin{array}{l}\text { Baseline } \\
\text { pts (\%) }\end{array}$ & $\begin{array}{l}1 \text { month } \\
\text { pts }(\%)\end{array}$ & $\begin{array}{l}3 \text { month } \\
\text { pts (\%) }\end{array}$ & $\begin{array}{l}6 \text { month } \\
\text { pts (\%) }\end{array}$ & p value \\
\hline $\begin{array}{l}\text { Absence of ED } \\
(22-25)\end{array}$ & 45 (45\%) & $\begin{array}{c}40(40 \%) \\
(p=0.477)\end{array}$ & $\begin{array}{c}43(43 \%) \\
(p=0.777)\end{array}$ & $\begin{array}{c}44(44 \%) \\
(p=0.888)\end{array}$ & $>0.05^{*}$ \\
\hline $\begin{array}{l}\text { Mild ED } \\
(17-21)\end{array}$ & 39 (39\%) & $\begin{array}{c}40(40 \%) \\
(p=0.886)\end{array}$ & $\begin{array}{c}42(42 \%) \\
(p=0.688)\end{array}$ & $\begin{array}{c}40(40 \%) \\
(p=0.886)\end{array}$ & $>0.05^{*}$ \\
\hline $\begin{array}{l}\text { Mild-moderate ED } \\
(12-16)\end{array}$ & 16 (16\%) & $\begin{array}{c}20(20 \%) \\
(p=0.464)\end{array}$ & $\begin{array}{c}15(15 \%) \\
(p=0846)\end{array}$ & $\begin{array}{c}16(16 \%) \\
(p=1)\end{array}$ & $>0.05^{*}$ \\
\hline $\begin{array}{l}\text { Moderate ED } \\
(8-11)\end{array}$ & - & - & - & - & - \\
\hline $\begin{array}{l}\text { Severe ED } \\
(5-7)\end{array}$ & - & - & - & - & - \\
\hline
\end{tabular}

ED: Erectile dysfunction;

*p value did not showed a statistically significant difference when baseline IIEF-5 was compared with post- biopsy IIEF-5 (1, 3 and 6 months from the procedure). 
equal to $11.6 \%$ and $0 \%$, respectively. Although in the last years number of repeat biopsies and/or needle cores (SPBx) has been supposed to temporary induce ED the literature data are conflicting. In fact, Fujita et al. (11) in 231 patients enrolled in active surveillance (AS) protocol for PCa found a correlation between number of repeat biopsy ( 3 or more) and erectile function; on the contrary, Hilton et al. (12) in 427 men on AS showed that sexual activity was not associated with biopsy exposure.

Recently, Klein et al. (13) in 198 patients submitted to 10 (155 cases) and 20 (53 cases) needle cores demonstrated that erectile function was transiently affected by prostate biopsy regardless of periprostatic nerve block and the number of cores.

In our series, to our knowledge the first that evaluated patients submitted to repeat transperineal SPBX under sedation, ED incidence did not significantly increased 1 , 3 and 6 months from biopsy; only 5/100 (5\%) men with a pre-biopsy normal sexual activity (IEEF-5 $>22$ ) referred at first month evaluation a mild ED (IEEF-5 score between 17 and 21) that disappeared 3-6 months later. Regarding our results some considerations should be done. Firstly, the true sexual activity of the couple administering a sexual questionnaire to the partners was not investigated. Secondly, we don't know if the transperineal biopsy approach and/or the absence of local anesthesia had a clinical impact on our results. Finally, in the absence a of control group we don't know if the onset of DE one month from SPBx (5\% of the cases) was really given by prostate biopsy.

In conclusion, repeat transperineal SPBx under sedation did not significantly worsened erectile function; the minimal risk of temporary post-biopsy ED could be previously discussed (not emphasised) with potent patients.

\section{References}

1. Pinkhasov GI, Lin YK, Palmerola R, et al Complications following prostate needle biopsy requiring or emergency department visitsexperience from 1000 consecutive cases. BJU Int. 2012; 110:369-374.
2. Pepe P, Aragona F. Morbidity following transperineal prostate biopsy in 3,000 patients submitted to 12 vs 18 vs more than 24 needle cores. Urology. 2013; 81:1142-1146.

3. Glaser AP, Novakic K, Helfand BT. The impact of prostate biopsy on urinary symptoms, erectile function, and anxiety. Curr Urol Rep. 2012; 13:447-454.

4. Zisman A, Leibovici D, Keinmann J, Siegel YI, Lindner A. The impact of prostate biopsy on patient well-being: a prospective study of pain, anxiety and erectile dysfunction. J Urol. 2001; 165:445-454.

5. Akbal C, Turker P, Tavukcu HH, et al. Erectile function in prostate cancer-free patients who underwent prostate saturation biopsy. Eur Urol. 2008; 53:540-544.

6. Palumbo F, Bettocchi C, Spilotros M, et al. A prospective study on patient's erectile function following transrectal ultrasound guided prostate biopsy. Arch Ital Urol Androl. 2010; 82:265-268.

7. Pepe P, Aragona F. Incidence of insignificant prostate cancer using free/total PSA: results of a case-finding protocol on 14453 patients. Prostate Cancer Prostatic Dis. 2010; 13:316-319.

8. Pepe P, Aragona F. Saturation prostate needle biopsy and prostate cancer detection at initial and repeat evaluation. Urology. 2007; 70:1131-1135.

9. Rosen RC, Cappelleri JC, Smith MD, et al. Development and evaluation of an abridged, 5-item version of the International Index of Erectile Function (IIEF-5) as a diagnostic tool for erectile dysfunction. Int J Impot Res. 1999; 11:319-326.

10. Tuncel A, Kirilmaz U, Nalcacioglu V, et al. The impact of transrectal prostate needle biopsy on sexuality in men and their female partners. Urology. 2008; 71:1128-1131.

11. Fujita K, Landis P, McNeil BK, Pavlovich CP. Serial prostate biopsies are associated with an increased risk of erectile dysfunction in men with prostate cancer on active surveillance. J Urol. 2009; 182:2664-2669.

12. Hilton JF, Blaschko SD, Whitson JM, et al. The impact of serial prostate biopsies on sexual function in men on active surveillance for prostate cancer. J Urol. 2012; 188:1252-1258.

13. Klein T, Palisaar RJ, Holz A, et al. The impact of prostate biopsy and periprostatic nerve block on erectile and voiding function: a prospective study. J Urol. 2010; 184:1447-1452.

\section{Correspondence}

Pietro Pepe, MD (Corresponding Author)

piepepe@hotmail.com

Francesco Pietropaolo, MD

Giuseppe Dibenedetto, MD

Francesco Aragona, MD

Urology Unit - Cannizzaro Hospital, via Messina 829 - Catania, Italy 